\title{
ALGEBRAIC INVARIANTS FOR RIGHT-ANGLED ARTIN GROUPS
}

\author{
STEFAN PAPADIMA AND ALEXANDER I. SUCIU ${ }^{1}$
}

\begin{abstract}
A finite simplicial graph $\Gamma$ determines a right-angled Artin group $G_{\Gamma}$, with generators corresponding to the vertices of $\Gamma$, and with a relation $v w=w v$ for each pair of adjacent vertices. We compute the lower central series quotients, the Chen quotients, and the (first) resonance variety of $G_{\Gamma}$, directly from the graph $\Gamma$.
\end{abstract}

\section{INTRODUCTION}

1.1. Right-angled Artin groups. Let $\Gamma=(\mathrm{V}, \mathrm{E})$ be a finite simplicial graph. To such a graph, there is associated a right-angled Artin group, $G_{\Gamma}$, with a generator $v$ for each vertex $v \in \mathrm{V}$, and with a commutator relation $v w=w v$ for each edge $\{v, w\} \in \mathrm{E}$.

From the graph $\Gamma$, one builds a simplicial complex, $\Delta_{\Gamma}$, called the flag complex, by filling in the complete subgraphs on $k$ vertices by $(k-1)$-simplices. In turn, the flag complex determines a CW-complex, $K_{\Gamma}$, called the cubical complex, by joining tori in the manner prescribed by $\Delta_{\Gamma}$ : the 0 -cell corresponds to the empty simplex, the 1-cells correspond to vertices $v \in \mathrm{V}$, the 2-cells correspond to edges $e \in \mathrm{E}$, etc.

Much is known about the topology of the cubical complex $K_{\Gamma}$, and its fundamental group, $G_{\Gamma}$. First of all, $K_{\Gamma}$ is an Eilenberg-MacLane $K\left(G_{\Gamma}, 1\right)$ space, see Charney and Davis [5] and Meier and VanWyk 21. The finiteness properties of the kernels of "diagonal" characters $G_{\Gamma} \rightarrow \mathbb{Z}$ have been related in a very precise way to the homotopical and homological properties of $\Delta_{\Gamma}$ by Bestvina and Brady 3. Finally, the geometric invariants of the type introduced by Bieri, Neumann, and Strebel 4 (invariants that hold subtle information about the finiteness properties of kernels of rational characters of $G_{\Gamma}$ ) have been determined by Meier, Meinert, and VanWyk [20].

1.2. Cohomology ring and formality. In this paper, we focus on a different set of invariants, mostly of an algebraic nature, for the right-angled Artin groups. The asphericity of $K_{\Gamma}$ leads to an identification of the cohomology ring $H^{*}\left(G_{\Gamma}, \mathbb{k}\right)$ with the exterior Stanley-Reisner ring $E / J_{\Gamma}$, where $E$ is the exterior algebra over $\mathbb{k}$, on generators $\left\{v^{*}\right\}_{v \in \mathrm{V}}$, and $J_{\Gamma}$ is the ideal generated by the monomials corresponding to non-edges.

A crucial observation, due to Fröberg [10, is that $E / J_{\Gamma}$ is a Koszul algebra. When combined with the fact that the group $G_{\Gamma}$ is 1-formal (cf. Kapovich and Millson [17]),

2000 Mathematics Subject Classification. Primary 20F36. Secondary 13F55, 20F14, 55P62, 57M07.

Key words and phrases. Graph, flag complex, cubical complex, right-angled Artin group, lower central series, holonomy Lie algebra, Chen Lie algebra, resonance variety.

${ }^{1}$ Partially supported by NSF grant DMS-0311142. 
this implies that the space $K_{\Gamma}$ is formal, see Proposition 3.3 In other words, all the rational homotopy theory of $K_{\Gamma}$ (embodied essentially in the Malcev completion of $G_{\Gamma}$ ) can be extracted, at least in principle, from $E / J_{\Gamma}$.

We give here a complete description of several invariants of a right-angled Artin group $G_{\Gamma}$ - the associated graded Lie algebra $\operatorname{gr}\left(G_{\Gamma}\right)$, the Chen $\operatorname{groups}_{\operatorname{gr}}\left(G_{\Gamma} / G_{\Gamma}^{\prime \prime}\right)$, and the first resonance variety $\mathcal{R}^{1}\left(G_{\Gamma} ; \mathbb{k}\right)$ - solely in terms of the graph $\Gamma$. Our approach will enable us to derive in 25] similar results for the Bestvina-Brady groups associated to $\Gamma$.

1.3. Lower central series and clique polynomial. We start by analyzing the graded Lie algebra $\operatorname{gr}(G)$ associated to a right-angled Artin group $G=G_{\Gamma}$. In Theorem 3.4. we show that $\operatorname{gr}(G)$ is isomorphic, as a graded Lie algebra, with $\mathfrak{H}(G)$, the holonomy Lie algebra defined from the reduced diagonal map $H_{2}(G) \rightarrow H_{1}(G) \wedge H_{1}(G)$. Furthermore, the lower central series quotients are torsion-free, with ranks $\phi_{k}$ given by

$$
\prod_{k=1}^{\infty}\left(1-t^{k}\right)^{\phi_{k}}=P_{\Gamma}(-t) .
$$

Here $P_{\Gamma}(t)=\sum_{k \geq 0} f_{k}(\Gamma) t^{k}$ is the clique polynomial of $\Gamma$, with coefficient $f_{k}(\Gamma)$ equal to the number of complete $k$-subgraphs of $\Gamma$ (in other words, $P_{\Gamma}(t)$ is the $f$ polynomial of the flag complex $\Delta_{\Gamma}$ ). This LCS formula is a manifestation of the Koszul duality between the universal enveloping algebra of $\mathfrak{H}(G)$ and the cohomology ring $H^{*}(G, \mathbb{k})=E / J$ (this duality was first noted by Shelton and Yuzvinsky [31]).

1.4. Chen groups and cut polynomial. Next, we determine the Chen Lie algebra, $\operatorname{gr}\left(G / G^{\prime \prime}\right)$. In Theorem 4.1 we show that the Chen groups of $G=G_{\Gamma}$ are also torsionfree, with ranks $\theta_{k}$ given by

$$
\sum_{k=2}^{\infty} \theta_{k} t^{k}=Q_{\Gamma}\left(\frac{t}{1-t}\right) .
$$

Here $Q_{\Gamma}(t)=\sum_{j \geq 2} c_{j}(\Gamma) t^{j}$ is the "cut polynomial" of $\Gamma$, with coefficient $c_{j}(\Gamma)$ equal to $\sum_{\mathrm{W} \subset \mathrm{V}:}|\mathrm{W}|=j \tilde{b}_{0}\left(\Gamma_{\mathrm{W}}\right)$, where $\tilde{b}_{0}\left(\Gamma_{\mathrm{W}}\right)$ is one less than the number of components of the full subgraph on $\mathrm{W}$.

The proof of (1.2) requires several steps. The first step, based on [23], is to identify, at least rationally, $\operatorname{gr}\left(G / G^{\prime \prime}\right)$ with $\mathfrak{H}(G) / \mathfrak{H}(G)^{\prime \prime}$. The next step, based on [12] (see also [30]), is to replace the computation of the Chen ranks by that of the Betti numbers in the linear strand of the free resolution of $E / J$ over $E$. A formula from [1] and [2] now expresses these Betti numbers in terms of the corresponding Betti numbers of the polynomial Stanley-Reisner ring, $S / I$, which in turn can be computed from the cut numbers of $\Gamma$ via a well-known formula of Hochster [16].

It is interesting to note that the Chen ranks $\theta_{k}$ often carry more information than the LCS ranks $\phi_{k}$. To illustrate this phenomenon, we give in 6.3 examples of graphs with the same clique polynomial, but different cut polynomials. 
1.5. Resonance varieties and cut sets. The numerical invariants $\phi_{k}(G)$ and $\theta_{k}(G)$ only reflect the additive structure of the respective graded Lie algebras, $\operatorname{gr}(G)$ and $\operatorname{gr}\left(G / G^{\prime \prime}\right)$. To capture more refined information, related to the Lie bracket, we turn to the resonance variety $\mathcal{R}^{1}(G ; \mathbb{k})$. First defined by Falk 9 ] in the context of hyperplane arrangements (over the field $\mathbb{k}=\mathbb{C}$ ), this subvariety of the affine space $H^{1}(G, \mathbb{k}$ ) depends only on the holonomy Lie algebra $\mathfrak{H}(G)$.

It turns out that the resonance variety of $G_{\Gamma}$ is determined by the cut sets of $\Gamma$. More precisely, we show in Theorem 5.5 that

$$
\mathcal{R}^{1}\left(G_{\Gamma}, \mathbb{k}\right)=\bigcup_{\substack{\mathrm{W} \subset \mathrm{V} \\ \Gamma_{\mathrm{W}} \text { disconnected }}} H_{\mathrm{W}},
$$

where $H_{\mathrm{W}}$ is the coordinate subspace spanned by $\mathrm{W}$ inside $H^{1}\left(G_{\Gamma}, \mathbb{k}\right)=\mathbb{k}^{|\mathrm{V}|}$. From this description, it becomes apparent that $\mathcal{R}^{1}\left(G_{\Gamma}, \mathbb{R}\right)$ is the complement of the BNS invariant $\Sigma^{1}\left(G_{\Gamma}\right)$.

To illustrate the strength of resonance, we give in $\$ 6.4$ examples of graphs sharing the same clique polynomial, and the same cut polynomial, but having different resonance varieties. The difference is rather subtle: both varieties have the same number of components, of the same dimension, but the respective intersection lattices are inequivalent.

1.6. Higher-dimensional cubical complexes. In [18, Kim and Roush defined simply-connected analogues of the cubical complex $K_{\Gamma}$. The CW-complexes $K_{\Gamma}^{q}(q \geq 1)$ are obtained by joining products of $(2 q+1)$-dimensional spheres in the manner prescribed by $\Delta_{\Gamma}$. For $q$ sufficiently large, these spaces are both formal and coformal. In 97 we compute the rational homology and homotopy groups of the loop spaces $\Omega K_{\Gamma}^{q}$, purely in terms of the clique polynomial of $\Gamma$.

We conclude with Example 7.3. which shows once again the usefulness of resonance varieties. Let $\Gamma$ and $\Gamma^{\prime}$ be two trees on the same number of vertices, but with different number of extremal vertices. Then $K_{\Gamma}^{q}$ and $K_{\Gamma^{\prime}}^{q}$ have the same rational homotopy groups, yet non-isomorphic homotopy Lie algebras. The difference in the respective Whitehead product structures can be traced back to the different number of components in $\mathcal{R}^{1}\left(G_{\Gamma}, \mathbb{Q}\right)$ and $\mathcal{R}^{1}\left(G_{\Gamma^{\prime}}, \mathbb{Q}\right)$.

\section{LIE ALGEBRAS AND FORMALITY}

2.1. Holonomy Lie algebra. Fix a ground $\operatorname{ring} R$, equal to either $\mathbb{Z}$ or a field $\mathbb{k}$. Let $A$ be a connected, graded algebra over $R$, with graded pieces $A^{i}, i \geq 0$. Assume $A^{1}$ is a finitely generated $R$-module (torsion-free if $R=\mathbb{Z}$ ), and $A^{1} \cdot A^{1}=0$, i.e., $a^{2}=0$, for all $a \in A^{1}$. Then, the multiplication map in degree 1 descends to an $R$-linear map $\mu: A^{1} \wedge A^{1} \rightarrow A^{2}$.

Let $\operatorname{Lie}\left(A_{1}\right)$ be the graded free Lie algebra on $A_{1}$, where $A_{i}=\left(A^{i}\right)^{\#}$ denotes the dual $R$-module. The holonomy Lie algebra of $A$, denoted $\mathfrak{H}(A)$, is the quotient of $\operatorname{Lie}\left(A_{1}\right)$ 
by the ideal generated by the image of the comultiplication map

$$
\nabla: A_{2}=\left(A^{2}\right)^{\# \stackrel{\mu^{\#}}{\longrightarrow}}\left(A^{1} \wedge A^{1}\right)^{\#} \cong A_{1} \wedge A_{1}=\operatorname{Lie}_{2}\left(A_{1}\right) .
$$

Note that $\mathfrak{H}(A)$ inherits a natural grading from Lie $\left(A_{1}\right)$; denote by $\mathfrak{H}(A)_{k}$ the $k$-th graded piece.

Now let $G$ be a finitely presented group. Then $A=H^{*}(G ; R)$ is a connected, graded-commutative $R$-algebra, with $A^{1}$ finitely generated (and torsion-free if $R=\mathbb{Z}$ ). If $R=\mathbb{Z}$, or $R=\mathbb{k}$ and char $\mathbb{k} \neq 2$, then automatically $A^{1} \cdot A^{1}=0$. Otherwise, we need to assume that the abelianization $H_{1}(G)$ is torsion-free, in which case it can be showed again that $A^{1} \cdot A^{1}=0$. In either case, we can define the holonomy Lie algebra of $G$, with coefficients in $R$, to be

$$
\mathfrak{H}_{R}(G)=\mathfrak{H}\left(H^{*}(G ; R)\right) \text {. }
$$

In the integral case, simply write $\mathfrak{H}(G)=\mathfrak{H}_{\mathbb{Z}}(G)$, and note that $\mathfrak{H}(G) \otimes \mathbb{Q}=\mathfrak{H}_{\mathbb{Q}}(G)$. Moreover, note that, in the case when $H_{1}(G)$ is torsion-free, the definition of $\mathfrak{H}(G)$ coincides with the one given in 23 .

2.2. Formality properties. In Appendix A of [27, Quillen associates, in a functorial way, a Malcev Lie algebra, $M_{G}$, to any group $G$ (see [23] for further details). A finitely presented group $G$ is said to be 1-formal if $M_{G}$ is isomorphic to the rational holonomy Lie algebra, $\mathfrak{H}_{\mathbb{Q}}(G)$, completed with respect to the bracket length filtration. Equivalently, $M_{G}$ is a quadratic Malcev Lie algebra.

Let $X$ be a finite-type, connected CW-complex. Then $X$ is formal, in the sense of Sullivan [33], if the rational homotopy type of $X$ is determined by the rational cohomology ring $H^{*}(X ; \mathbb{Q})$. As shown by Sullivan, the fundamental group of a formal space is 1-formal. The 1-formality of $\pi_{1}(X)$ is not enough to insure the formality of $X$; indeed, simply-connected, non-formal spaces are easily constructed. Nevertheless, we have the following positive result in this direction.

Recall that a connected, graded algebra $A$ over a field $\mathbb{k}$ is called a Koszul algebra if $\operatorname{Tor}_{i}^{A}(\mathbb{k}, \mathbb{k})_{j}=0$, for all $i \neq j$. A necessary condition is that $A$ be the quotient of a free algebra on generators in degree 1 by an ideal $I$ generated in degree 2 . A sufficient condition is that $I$ admit a quadratic Gröbner basis.

Proposition 2.1. Let $X$ be a finite-type, connected $C W$-complex. Suppose $H^{*}(X ; \mathbb{Q})$ is a Koszul algebra, and $G=\pi_{1}(X)$ is 1-formal. Then $X$ is a formal space.

Proof. By assumption, $M_{G} \cong \widehat{\mathfrak{H}_{\mathbb{Q}}(G)}$. This condition can be reinterpreted in terms of Sullivan's minimal models, as follows:

$$
\mathfrak{M}_{1}\left(\Omega_{\mathrm{dR}}^{*} X, d\right) \cong \mathfrak{M}_{1}\left(H^{*}(X ; \mathbb{Q}), d=0\right),
$$

where $\mathfrak{M}(A, d)$ denotes the minimal model of a differential graded algebra $(A, d)$, and $\mathfrak{M}_{1}$ denotes the subalgebra generated by the degree 1 part of $\mathfrak{M}$, see 33 . On the other hand, formality of $X$ means that

$$
\mathfrak{M}\left(\Omega_{\mathrm{dR}}^{*} X, d\right) \cong \mathfrak{M}\left(H^{*}(X ; \mathbb{Q}), d=0\right) .
$$


The Koszulness assumption on $H^{*}(X ; \mathbb{Q})$ implies that $\mathfrak{M}\left(\Omega_{\mathrm{dR}}^{*} X, d\right)=\mathfrak{M}_{1}\left(\Omega_{\mathrm{dR}}^{*} X, d\right)$ and $\mathfrak{M}\left(H^{*}(X ; \mathbb{Q}), d=0\right)=\mathfrak{M}_{1}\left(H^{*}(X ; \mathbb{Q}), d=0\right)$. This observation (which follows readily from [26, Proposition 5.2]), finishes the proof.

2.3. Lower central series. Let $G$ be a group. The lower central series of $G$ is the sequence of normal subgroups $\left\{\gamma_{k} G\right\}_{k \geq 1}$, defined inductively by $\gamma_{1} G=G, \gamma_{2} G=G^{\prime}$, and $\gamma_{k+1} G=\left(\gamma_{k} G, G\right)$, where $(x, y)=x y x^{-1} y^{-1}$. Observe that the successive quotients $\gamma_{k} G / \gamma_{k+1} G$ are abelian groups. The direct sum of these quotients,

$$
\operatorname{gr}(G)=\bigoplus_{k \geq 1} \gamma_{k} G / \gamma_{k+1} G
$$

is the associated graded Lie algebra of $G$. The Lie bracket $[x, y]$ is induced from the group commutator, while the grading is given by bracket length.

Assume $G$ is finitely generated. Since $\operatorname{gr}(G)$ is generated as a Lie algebra by the degree 1 piece, $\operatorname{gr}_{1}(G)=G / G^{\prime}$, all the graded pieces $\operatorname{gr}_{k}(G)=\gamma_{k} G / \gamma_{k+1} G$ are finitely generated abelian groups. If, moreover, $G$ is 1 -formal, then

$$
\operatorname{gr}(G) \otimes \mathbb{Q} \cong \mathfrak{H}_{\mathbb{Q}}(G)
$$

as graded Lie algebras, as follows from Appendix A in [27.

Assume now $G$ is finitely presented, and $H_{1}(G)$ is torsion-free. Then, the canonical projection $\operatorname{Lie}\left(H_{1}(G)\right) \rightarrow \operatorname{gr}(G)$ factors through an epimorphism $\Psi_{G}: \mathfrak{H}(G) \rightarrow \operatorname{gr}(G)$. If, moreover, $G$ is 1 -formal and $\mathfrak{H}(G)$ is torsion-free, then the map $\Psi_{G}$ yields an isomorphism of (integral) graded Lie algebras,

$$
\Psi_{G}: \mathfrak{H}(G) \stackrel{\cong}{\longrightarrow} \operatorname{gr}(G) .
$$

The Chen Lie algebra of a group $G$ is the associated graded Lie algebra of its maximal metabelian quotient, $G / G^{\prime \prime}$. Assume $G$ is 1-formal. Then, as shown in 23],

$$
\operatorname{gr}\left(G / G^{\prime \prime}\right) \otimes \mathbb{Q} \cong \mathfrak{H}_{\mathbb{Q}}(G) / \mathfrak{H}_{\mathbb{Q}}(G)^{\prime \prime}
$$

Assume now $G$ is finitely presented, and $H_{1}(G)$ is torsion-free. Then, the map $\Psi_{G}$ descends to an epimorphism of graded Lie algebras, $\Psi_{G}^{(2)}: \mathfrak{H}(G) / \mathfrak{H}(G)^{\prime \prime} \rightarrow \operatorname{gr}\left(G / G^{\prime \prime}\right)$. If, moreover, $G$ is 1 -formal and $\mathfrak{H}(G) / \mathfrak{H}(G)^{\prime \prime}$ is torsion-free, then, by [23, Theorem 1.2], the map $\Psi_{G}^{(2)}$ yields an isomorphism

$$
\Psi_{G}^{(2)}: \mathfrak{H}(G) / \mathfrak{H}(G)^{\prime \prime} \stackrel{\cong}{\longrightarrow} \operatorname{gr}\left(G / G^{\prime \prime}\right) .
$$

Of particular interest is the determination of the LCS ranks, $\phi_{k}(G)=\operatorname{rank}_{k} \operatorname{gr}_{k}(G)$ and Chen ranks, $\theta_{k}(G)=\operatorname{rankgr}_{k}\left(G / G^{\prime \prime}\right)$. Note that $\phi_{1}(G)=\theta_{1}(G)=\operatorname{rank} H_{1}(G)$; moreover, $\phi_{2}(G)=\theta_{2}(G), \phi_{3}(G)=\theta_{3}(G)$, and $\phi_{k}(G) \geq \theta_{k}(G)$, for $k \geq 4$.

Example 2.2. Let $F_{n}$ be the free group of rank $n$. The associated graded Lie algebra $\operatorname{gr}\left(F_{n}\right)$ is isomorphic to the free Lie algebra $\mathbf{L}_{n}=\operatorname{Lie}\left(\mathbb{Z}^{n}\right)$, while $\operatorname{gr}\left(F_{n} / F_{n}^{\prime \prime}\right) \cong \mathbf{L}_{n} / \mathbf{L}_{n}^{\prime \prime}$. 
The LCS ranks are given by Witt's formula: $\phi_{k}\left(F_{n}\right)=\frac{1}{k} \sum_{d \mid k} \mu(d) n^{k / d}$, or, equivalently,

$$
\prod_{k=1}^{\infty}\left(1-t^{k}\right)^{\phi_{k}\left(F_{n}\right)}=1-n t,
$$

while the Chen ranks are given by K.-T. Chen's formula: $\theta_{k}\left(F_{n}\right)=(k-1)\left(\begin{array}{c}n+k-2 \\ k\end{array}\right)$, for $k \geq 2$, or, equivalently,

$$
\sum_{k=2}^{\infty} \theta_{k}\left(F_{n}\right) t^{k}=1-\frac{1-n t}{(1-t)^{n}},
$$

2.4. Homological algebra interpretation. Let $X$ be a finite-type, connected CW complex, with fundamental group $G=\pi_{1}(X)$ and rational cohomology $\operatorname{ring} A=$ $H^{*}(X ; \mathbb{Q})$. Assume $G$ is a 1 -formal group. Using the isomorphism (2.4), Corollary 7.16 from Halperin and Stasheff [15] yields:

$$
\phi_{k}(G)=\operatorname{dim}_{\mathbb{Q}} \operatorname{Prim} \operatorname{Ext}_{A}^{k}(\mathbb{Q}, \mathbb{Q})_{k} .
$$

Here, Prim $\operatorname{Ext}_{A}(\mathbb{Q}, \mathbb{Q})$ denotes the primitives in the bigraded Hopf Ext-algebra of $A$, where the upper degree is the resolution degree, and the lower degree is the internal degree.

We conclude this section with an analogue of formula (2.10) for the Chen ranks. The key tool is the following result of Fröberg and Löfwall 12 .

Proposition 2.3. Let $A$ be a graded algebra over a field $\mathbb{k}$, with $A^{1}$ finite-dimensional and $A^{1} \cdot A^{1}=0$. Assume $A$ is generated in degree 1 , i.e, the canonical map from the exterior algebra $E=\bigwedge A^{1}$ to $A$ is surjective. Then:

$$
\left(\mathfrak{H}(A)^{\prime} / \mathfrak{H}(A)^{\prime \prime}\right)_{k}^{\#}=\operatorname{Tor}_{k-1}^{E}(A, \mathbb{k})_{k}, \quad \text { for } k \geq 2 .
$$

Proof. In the case when char $\mathbb{k} \neq 2$, the conclusion follows directly from Theorem 4.1(ii) in [12. When char $\mathbb{k}=2$, the minimal model proof of that Theorem still works, given our assumption that $A^{1} \cdot A^{1}=0$.

Corollary 2.4. Let $G$ be a finitely presented, 1-formal group. Let $A$ be a gradedcommutative $\mathbb{Q}$-algebra, such that $\operatorname{dim} A^{1}<\infty$ and the map $E=\wedge A^{1} \rightarrow A$ is surjective. Assume $\mathfrak{H}(A)=\mathfrak{H}_{\mathbb{Q}}(G)$. Then:

$$
\theta_{k}(G)=\operatorname{dim}_{\mathbb{Q}} \operatorname{Tor}_{k-1}^{E}(A, \mathbb{Q})_{k}, \quad \text { for } k \geq 2 .
$$

Proof. Follows from (2.6) and Proposition 2.3.

Given a group $G$ as above, an algebra $A$ with the required properties can be constructed as follows. Let $K=\operatorname{ker}\left(\mu: H^{1}(G ; \mathbb{Q}) \wedge H^{1}(G ; \mathbb{Q}) \rightarrow H^{2}(G, \mathbb{Q})\right)$, and $E=$ $\bigwedge H^{1}(G ; \mathbb{Q})$. Set $A=E /\left(K+E^{\geq 3}\right)$. It is then readily checked that $\mathfrak{H}(A)=\mathfrak{H}_{\mathbb{Q}}(G)$.

In the case when $G$ is the fundamental group of the complement of a complex hyperplane arrangement, and $A$ is the Orlik-Solomon algebra of the arrangement, a different proof of formula (2.12) was given in 30. 


\section{Homology And lower Central SERIES}

3.1. The cubical complex. By a graph $\Gamma=\left(\mathrm{V}_{\Gamma}, \mathrm{E}_{\Gamma}\right)$ we mean a loopless, finite graph without multiple edges (i.e., a one-dimensional, finite simplicial complex), with vertex set $\mathrm{V}_{\Gamma}$ and edge set $\mathrm{E}_{\Gamma} \subset\left(\begin{array}{c}\mathrm{V}_{\Gamma} \\ 2\end{array}\right)$. To such a graph, there corresponds a right-angled Artin group (or, graph group), denoted $G_{\Gamma}$, with presentation

$$
\left.G_{\Gamma}=\left\langle v \in \mathrm{V}_{\Gamma}\right| u v=v u \text { if }\{u, v\} \in \mathrm{E}_{\Gamma}\right\rangle .
$$

The flag complex of $\Gamma$, denoted $\Delta_{\Gamma}$, is the maximal simplicial complex with 1-skeleton equal to $\Gamma$. The $k$-simplices of $\Delta_{\Gamma}$ are the $(k+1)$-cliques of $\Gamma$, that is, the complete subgraphs on $(k+1)$ vertices.

Let $K_{\Gamma}$ be the associated cubical complex, obtained by joining tori in the manner prescribed by $\Delta_{\Gamma}$. More precisely, for each simplex $\sigma$ of $\Delta_{\Gamma}$, let $T_{\sigma}$ be the torus formed by identifying parallel faces of a $(|\sigma|+1)$-cube $\left(T_{\emptyset}\right.$ is a point); then:

$$
K_{\Gamma}=\bigcup_{\sigma \in \Delta_{\Gamma}} T_{\sigma} /\left(T_{\sigma} \cap T_{\sigma^{\prime}}=T_{\tau} \text { if } \sigma \cap \sigma^{\prime}=\tau\right) .
$$

In other words, if $\left(S^{1}\right)^{\times n}$ is the torus of dimension $n=\left|\mathrm{V}_{\Gamma}\right|$, with the usual CWdecomposition, then $K_{\Gamma}$ is the subcomplex obtained by deleting the cells corresponding to the non-faces of $\Delta_{\Gamma}$.

Note that $H_{k}\left(K_{\Gamma}\right)$ is free abelian, of rank equal to the number, $f_{k}(\Gamma)$, of $k$-cliques in $\Gamma$ (where $f_{0}(\Gamma)=1$ ). In particular, the Poincaré polynomial of the cubical complex, $\operatorname{Poin}\left(K_{\Gamma}, t\right)=\sum_{k \geq 0} b_{k}\left(K_{\Gamma}\right) t^{k}$, equals the clique polynomial of the graph, $P_{\Gamma}(t)=$ $\sum_{k \geq 0} f_{k}(\Gamma) t^{k}$

It is readily seen that the fundamental group of $K_{\Gamma}$ is the Artin group $G_{\Gamma}$. In fact, $K_{\Gamma}$ is an Eilenberg-MacLane space of type $K\left(G_{\Gamma}, 1\right)$, see [5], 21].

Example 3.1. Let $\Gamma_{1}$ and $\Gamma_{2}$ be two graphs (on distinct vertex sets). The join of the two graphs, $\Gamma=\Gamma_{1} * \Gamma_{2}$, is the graph with vertex set $\mathrm{V}=\mathrm{V}_{1} \cup \mathrm{V}_{2}$ and edge set $\mathrm{E}=\mathrm{E}_{1} \cup \mathrm{E}_{2} \cup\left\{\left\{v_{1}, v_{2}\right\} \mid v_{1} \in \mathrm{V}_{1}\right.$ and $\left.v_{2} \in \mathrm{V}_{2}\right\}$. Clearly, $G_{\Gamma}=G_{\Gamma_{1}} \times G_{\Gamma_{2}}$ and $K_{\Gamma}=K_{\Gamma_{1}} \times K_{\Gamma_{2}}$.

For instance, let $\Gamma=K_{n}$ be the complete graph on $n$ vertices (the iterated join of $n$ graphs on a single vertex). In this case, $\Delta_{\Gamma}$ is the $(n-1)$-simplex, while $K_{\Gamma}$ is the $n$-torus, with fundamental group $G_{\Gamma}=\mathbb{Z}^{n}$.

Example 3.2. Suppose $\Gamma=\Gamma_{1} \amalg \Gamma_{2}$ is the disjoint union of two subgraphs. Then $G_{\Gamma}=G_{\Gamma_{1}} * G_{\Gamma_{2}}$ and $K_{\Gamma}=K_{\Gamma_{1}} \vee K_{\Gamma_{2}}$.

For instance, let $\Gamma=\bar{K}_{n}$ be the complement of $K_{n}$ (note that $\bar{K}_{n}$ is the graph on $n$ vertices, with empty edge set). In this case, $\Delta_{\Gamma}=\bar{K}_{n}$, while $K_{\Gamma}$ is the wedge of $n$ circles, with fundamental group $G_{\Gamma}=F_{n}$.

For each subset $\mathrm{W} \subset \mathrm{V}$, let $\Gamma_{\mathrm{W}}$ be the full subgraph of $\Gamma$ on vertex set $\mathrm{W}$. Let $G_{\mathrm{W}}=G_{\Gamma_{\mathrm{W}}}$ be the corresponding right-angled Artin group, and let $K_{\mathrm{W}}=K_{\Gamma_{\mathrm{W}}}$ be the corresponding cubical complex. The inclusion $\mathrm{W} \subset \mathrm{V}$ gives rise to a cellular inclusion map $j_{\mathrm{W}}: K_{\mathrm{W}} \rightarrow K_{\Gamma}$. The induced homomorphism, $\left(j_{\mathrm{W}}\right)_{\#}: G_{\mathrm{W}} \rightarrow G_{\Gamma}$, is a split 
injection, with retract $G_{\Gamma} \rightarrow G_{\mathrm{W}}$ given on generators by $v \mapsto v$ if $v \in \mathrm{W}$, and $v \mapsto 1$ otherwise.

3.2. Cohomology ring and formality. Fix a labeling of the vertices of the graph $\Gamma$, and write $\mathrm{V}_{\Gamma}=\left\{v_{1}, \ldots, v_{n}\right\}$. As first noted by Kim and Roush [18, the cohomology ring of $K_{\Gamma}$ is the quotient of $E$, the exterior algebra on generators $v_{1}^{*}, \ldots, v_{n}^{*}$ in degree 1 , modulo the ideal $J_{\Gamma}$ generated by the monomials $v_{i}^{*} v_{j}^{*}$ for which $\left\{v_{i}, v_{j}\right\}$ is not an edge of $\Gamma$. In other words,

$$
H^{*}\left(K_{\Gamma}\right)=E / J_{\Gamma}
$$

is the exterior Stanley-Reisner ring of $\Gamma$ (or, of the flag complex $\Delta_{\Gamma}$ ). For example, if $\Gamma=K_{n}$, then $H^{*}\left(K_{\Gamma}\right)=E$, while if $\Gamma=\bar{K}_{n}$, then $H^{*}\left(K_{\Gamma}\right)=E / \mathfrak{m}^{2}$, where $\mathfrak{m}$ is the ideal generated by $v_{1}^{*}, \ldots, v_{n}^{*}$.

As noted by Fröberg [10] (and reproved by Shelton and Yuzvinsky [31]), the set $\left\{v_{i}^{*} v_{j}^{*} \mid\left\{v_{i}, v_{j}\right\} \notin \mathrm{E}_{\Gamma}\right\}$ is a quadratic Gröbner basis for $J_{\Gamma}$; thus, $H^{*}\left(K_{\Gamma}, \mathbb{k}\right)$ is a Koszul algebra, for any coefficient field $\mathbb{k}$. Consequently, $K_{\Gamma}$ is not only a $K(\pi, 1)$-space, but also a rational $K(\pi, 1)$-space, i.e., its Bousfield-Kan rationalization is aspherical, cf. [26].

As shown by Kapovich and Millson in [17, Theorem 16.10], all Artin groups are 1formal; in particular, the right-angled Artin groups $G_{\Gamma}$ are 1-formal. Using Proposition 2.1. we obtain:

Proposition 3.3. Let $K_{\Gamma}$ be the cubical complex associated to a graph $\Gamma$. Then $K_{\Gamma}$ is a formal space.

3.3. Associated graded Lie algebra. We are now ready to compute the graded Lie algebra associated to the lower central series filtration of a right-angled Artin group.

Theorem 3.4. Let $\Gamma=(\mathrm{V}, \mathrm{E})$ be a finite graph. Let $G_{\Gamma}$ be the corresponding rightangled Artin group, with associated graded Lie algebra $\operatorname{gr}\left(G_{\Gamma}\right)$. Then:

(1) The holonomy Lie algebra of $G_{\Gamma}$ has presentation

$$
\mathfrak{H}\left(G_{\Gamma}\right)=\operatorname{Lie}(\mathrm{V}) /([v, w]=0 \text { if }\{v, w\} \in \mathrm{E}) .
$$

(2) The graded abelian group $\mathfrak{H}\left(G_{\Gamma}\right)$ is torsion-free.

(3) The canonical projection $\Psi: \mathfrak{H}\left(G_{\Gamma}\right) \rightarrow \operatorname{gr}\left(G_{\Gamma}\right)$ is an isomorphism of graded Lie algebras over $\mathbb{Z}$.

(4) The ranks of the graded pieces of $\operatorname{gr}\left(G_{\Gamma}\right)$ are given by

$$
\prod_{k=1}^{\infty}\left(1-t^{k}\right)^{\phi_{k}\left(G_{\Gamma}\right)}=P_{\Gamma}(-t),
$$

where $P_{\Gamma}(t)=\sum_{k \geq 0} f_{k}(\Gamma) t^{k}$ is the clique polynomial of the graph $\Gamma$.

Proof. Part (11) follows from the definition of the holonomy Lie algebra of $G=G_{\Gamma}$, and the identification of $H^{*}(G)$ with the exterior Stanley-Reisner ring of $\Gamma$. 
To prove (2), it is enough to show that the Hilbert series of $\mathfrak{H}(G) \otimes \mathbb{k}$ does not depend on the field $\mathbb{k}$. By the Poincaré-Birkhoff-Witt theorem, it is enough to show that the Hilbert series of the universal enveloping algebra $U \mathfrak{H}_{\mathbb{k}}(G)$ does not depend on $\mathbb{k}$. Now, as noted by Shelton and Yuzvinsky in $31, U \mathfrak{H}_{\mathbb{k}}(G)=A_{\mathbb{k}}^{!}$, where $A_{\mathbb{k}}=H^{*}(G ; \mathbb{k})$, and $A^{!}$is the Koszul dual of a quadratic algebra $A$. But $A_{\mathbb{k}}$ is a Koszul algebra, and so $\operatorname{Hilb}\left(A_{\mathbb{k}}^{!}, t\right)=\operatorname{Hilb}\left(A_{\mathbb{k}},-t\right)^{-1}$ by Koszul duality. Finally, note that $\operatorname{Hilb}\left(A_{\mathbb{k}}, t\right)=P_{\Gamma}(t)$ is independent of $\mathbb{k}$.

Part (3) follows from Part (2), together with the 1-formality of $G$ and formula (2.5).

Part (4) follows from Part (3), together with the proof of Part (2).

\section{Stanley-Reisner Rings And Chen groups}

4.1. Stanley-Reisner rings. Let $\Gamma$ be a finite graph. Put a total order on the vertex set, and write $\mathrm{V}_{\Gamma}=\left\{v_{1}, \ldots, v_{n}\right\}$. As mentioned 3.2 the cohomology ring $H^{*}\left(G_{\Gamma} ; \mathbb{k}\right)$ is isomorphic to the exterior Stanley-Reisner ring, $E / J_{\Gamma}$, where $E=\bigwedge_{\mathbb{k}}\left(v_{1}^{*}, \ldots, v_{n}^{*}\right)$ and $J_{\Gamma}=$ ideal $\left\langle v_{i}^{*} v_{j}^{*} \mid\left\{v_{i}, v_{j}\right\} \notin \mathrm{E}_{\Gamma}\right\rangle$. The Hilbert series of this graded ring equals the clique polynomial: $\operatorname{Hilb}\left(E / J_{\Gamma}, t\right)=P_{\Gamma}(t)$,

Associated to $\Gamma$ there is also the (polynomial) Stanley-Reisner ring, $S / I_{\Gamma}$, where $S=\mathbb{k}\left[x_{1}, \ldots, x_{n}\right]$ and $I_{\Gamma}=$ ideal $\left\langle x_{i} x_{j} \mid\left\{v_{i}, v_{j}\right\} \notin \mathrm{E}_{\Gamma}\right\rangle$. As shown in 32, the Hilbert series of this graded ring is also determined by the clique polynomial:

$$
\operatorname{Hilb}\left(S / I_{\Gamma}, t\right)=P_{\Gamma}\left(\frac{t}{1-t}\right) .
$$

A finitely generated, $\mathbb{N}^{n}$-graded module $M$ over $A=S$ or $E$ has a minimal resolution by free $A$-modules and multi-homogeneous $A$-linear maps; such a resolution, $F_{*} \rightarrow M$, is unique up to isomorphism. Denote by $\beta_{i, a}^{A}(M)$ the number of basis elements in $F_{i}$ that are homogeneous of multi-degree $a=\left(a_{1}, \ldots, a_{n}\right)$, with $a_{j} \geq 0$. By minimality of the resolution, $\beta_{i, a}^{A}(M)=\operatorname{dim}_{\mathbb{k}} \operatorname{Tor}_{i}^{A}(M, \mathbb{k})_{a}$.

The multi-graded Poincaré series of the two Stanley-Reisner rings are related by the following remarkable formula (see [2, (6.4)], 11, Proposition 2.1], or [8, Corollary 5.7]):

$$
\sum_{i \geq 0} \sum_{a \in \mathbb{N}^{n}} \beta_{i, a}^{E}\left(E / J_{\Gamma}\right) t^{i} u^{a}=\sum_{i \geq 0} \sum_{a \in \mathbb{N}^{n}} \beta_{i, a}^{S}\left(S / I_{\Gamma}\right) \frac{t^{i} u^{a}}{\prod_{j \in \operatorname{supp}(a)}\left(1-t u_{j}\right)} .
$$

Moreover, since $I_{\Gamma}$ is a square-free monomial ideal, all the nonzero, multi-graded Betti numbers $\beta_{i, a}^{S}\left(S / I_{\Gamma}\right)$ are also square-free, in the sense that $a_{j}=0$ or 1 ; see Hochster [16.

4.2. Chen Lie algebra of $G_{\Gamma}$. Before stating our next result, we need to establish some terminology. For a graph $\Gamma$ on vertex set $\mathrm{V}$, let $\kappa(\Gamma)$ be the connectivity of $\Gamma$, that is, the maximum integer $r$ so that, for any subset $\mathrm{W} \subset \mathrm{V}$ with $|\mathrm{W}|<r$, the full subgraph of $\Gamma$ on $\mathrm{V} \backslash \mathrm{W}$ is connected. Also, let $\tilde{b}_{0}(\Gamma)=\operatorname{rank} \widetilde{H}_{0}(\Gamma)$ be the number of components of $\Gamma$ minus 1 . 
For each $j \geq 1$, define the $j$-th cut number of $\Gamma$ to be:

$$
c_{j}(\Gamma)=\sum_{\mathrm{W} \subset \mathrm{V}:|\mathrm{W}|=j} \tilde{b}_{0}\left(\Gamma_{\mathrm{W}}\right) .
$$

Note that $c_{1}(\Gamma)=0$, and also $c_{j}(\Gamma)=0$, if $j>|\mathrm{V}|-\kappa(\Gamma)$. We are thus led to define the cut polynomial of $\Gamma$ as

$$
Q_{\Gamma}(t)=\sum_{j=2}^{|\mathrm{V}|-\kappa(\Gamma)} c_{j}(\Gamma) t^{j} .
$$

Theorem 4.1. Let $\Gamma$ be a finite graph. Let $G_{\Gamma}$ be the corresponding right-angled Artin group, with Chen Lie algebra $\operatorname{gr}\left(G_{\Gamma} / G_{\Gamma}^{\prime \prime}\right)$. Then:

(1) The graded abelian group $\mathfrak{H}\left(G_{\Gamma}\right) / \mathfrak{H}\left(G_{\Gamma}\right)^{\prime \prime}$ is torsion-free.

(2) The natural map $\Psi^{(2)}: \mathfrak{H}\left(G_{\Gamma}\right) / \mathfrak{H}\left(G_{\Gamma}\right)^{\prime \prime} \rightarrow \operatorname{gr}\left(G_{\Gamma} / G_{\Gamma}^{\prime \prime}\right)$ is an isomorphism of graded Lie algebras over $\mathbb{Z}$.

(3) The ranks of the graded pieces of $\operatorname{gr}\left(G_{\Gamma} / G_{\Gamma}^{\prime \prime}\right)$ are given by

$$
\sum_{k=2}^{\infty} \theta_{k}\left(G_{\Gamma}\right) t^{k}=Q_{\Gamma}\left(\frac{t}{1-t}\right),
$$

where $Q_{\Gamma}(t)=\sum_{j \geq 2} c_{j}(\Gamma) t^{j}$ is the cut polynomial of $\Gamma$.

Proof. Let $\mathbb{k}$ be a field. Applying Proposition 2.3 to the exterior Stanley-Reisner ring over $\mathbb{k}$, we obtain:

$$
\operatorname{dim}_{\mathbb{k}}\left(\mathfrak{H}_{\mathbb{k}}\left(G_{\Gamma}\right) / \mathfrak{H}_{\mathbb{k}}\left(G_{\Gamma}\right)^{\prime \prime}\right)_{k}=\beta_{k-1, k}^{E}\left(E / J_{\Gamma}\right), \text { for } k \geq 2 .
$$

Using formula (4.2), we find:

$$
\sum_{k \geq 2} \beta_{k-1, k}^{E}\left(E / J_{\Gamma}\right) t^{k}=\sum_{i \geq 1} \beta_{i, i+1}^{S}\left(S / I_{\Gamma}\right)\left(\frac{t}{1-t}\right)^{i+1} .
$$

A well-known formula of Hochster [16, Theorem 5.1] gives:

$$
\beta_{i, i+1}^{S}\left(S / I_{\Gamma}\right)=\sum_{\mathrm{W} \subset \mathrm{V}:|\mathrm{W}|=i+1} \operatorname{dim}_{\mathbb{k}} \widetilde{H}_{0}\left(\Gamma_{\mathrm{W}}, \mathbb{k}\right)=c_{i+1}(\Gamma)
$$

compare with [29, Proposition 2.1]. Combining (4.6), (4.7), and (4.8), we see that $\operatorname{dim}_{\mathbb{k}}\left(\mathfrak{H}\left(G_{\Gamma}\right) / \mathfrak{H}\left(G_{\Gamma}\right)^{\prime \prime}\right)_{k} \otimes \mathbb{k}$ is independent of $\mathbb{k}$. This finishes the proof of Part (11).

Part (2) follows from Part (11), together with the 1-formality of $G_{\Gamma}$ and formula (2.7). Part (3), follows from Part (2), together with formulas (4.6)-(4.8).

\section{Cut SETS AND Resonance VARIETiEs}

5.1. Resonance variety of an algebra. Let $A$ be a connected, graded algebra over a field $\mathbb{k}$. Assume $0<\operatorname{dim} A^{1}<\infty$, and $A^{1} \cdot A^{1}=0$. Then, for each $a \in A^{1}$, right-multiplication by $a$ defines a cochain complex

$$
(A, a): \quad A^{0} \stackrel{a}{\longrightarrow} A^{1} \stackrel{a}{\longrightarrow} A^{2} .
$$


The (first) resonance variety of $A$ is the locus of points $a$ in the affine space $A^{1}$ where this complex fails to be exact in the middle:

$$
\mathcal{R}^{1}(A)=\left\{a \in A^{1} \mid H^{1}(A, a) \neq 0\right\} .
$$

Clearly, $\mathcal{R}^{1}(A)$ is a homogeneous algebraic variety; in particular, it contains $0 \in A^{1}$.

Note that a nonzero element $a \in A^{1}$ belongs to $\mathcal{R}^{1}(A)$ if and only if there is an element $a^{\prime} \in A^{1}$ such that

$$
a \wedge a^{\prime} \neq 0 \text { in } A^{1} \wedge A^{1} \text { and } \mu\left(a \wedge a^{\prime}\right)=0 \text { in } A^{2},
$$

where $\mu: A^{1} \wedge A^{1} \rightarrow A^{2}$ is the multiplication map. In particular, $\mathcal{R}^{1}(A)$ depends only on the corestriction of $\mu$ to its image, and hence, only on the holonomy Lie algebra $\mathfrak{H}(A)$.

Lemma 5.1. Let $\varphi: A \rightarrow B$ be a morphism of graded algebras. If $\varphi_{1}: A^{1} \rightarrow B^{1}$ is injective, then $\varphi_{1}\left(\mathcal{R}^{1}(A)\right) \subset \mathcal{R}^{1}(B)$.

Proof. We have the following commuting diagram:

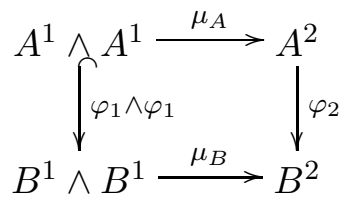

Let $a$ be a nonzero element in $\mathcal{R}^{1}(A) \subset A^{1}$. Pick $a^{\prime} \in A^{1}$ such that (5.3) holds. Then $\varphi_{1}(a) \wedge \varphi_{1}\left(a^{\prime}\right) \neq 0$, by injectivity of $\varphi_{1}$, and $\mu_{B}\left(\varphi_{1}(a) \wedge \varphi_{1}\left(a^{\prime}\right)\right)=0$, by commutativity of the diagram. Hence, $\varphi_{1}(a)$ belongs to $\mathcal{R}^{1}(B)$.

In particular, if $\varphi: A \rightarrow B$ is an isomorphism of graded algebras, then the linear isomorphism $\varphi_{1}: A^{1} \rightarrow B^{1}$ restricts to an isomorphism $\mathcal{R}^{1}(A) \rightarrow \mathcal{R}^{1}(B)$ between the corresponding resonance varieties.

Lemma 5.2. Suppose the vector space $A^{1}$ decomposes as a nontrivial direct sum $U_{1} \oplus$ $U_{2}$, with $\mu_{A}\left(u_{1} \wedge u_{2}\right)=0$ for all $u_{1} \in U_{1}$ and $u_{2} \in U_{2}$. Then $\mathcal{R}^{1}(A)=A^{1}$.

Proof. Let $Z=\left\{a \in A^{1} \mid a=u_{1}+u_{2}\right.$, with $u_{i} \in U_{i}$ and $\left.u_{i} \neq 0\right\}$. Clearly, $Z$ is a nonempty, Zariski open subset of $A^{1}$, so it suffices to show that $Z \subset \mathcal{R}^{1}(A)$.

Let $a=u_{1}+u_{2} \in Z$. Then $a \wedge u_{2}=u_{1} \wedge u_{2} \neq 0$ and $\mu_{A}\left(a \wedge u_{2}\right)=\mu_{A}\left(u_{1} \wedge u_{2}\right)=0$. Thus, $a$ belongs to $\mathcal{R}^{1}(A)$.

5.2. Resonance variety of a group. Let $G$ be a finitely presented group, with $H_{1}(G)$ nonzero and torsion-free. Then, the cohomology $\operatorname{ring} A=H^{*}(G ; \mathbb{k})$ satisfies the condition $a^{2}=0$ for all $a \in A^{1}$, and so the resonance variety of $G$ (over the field $\mathbb{k}$ ) can be defined as

$$
\mathcal{R}^{1}(G, \mathbb{k})=\mathcal{R}^{1}\left(H^{*}(G ; \mathbb{k})\right) .
$$

Lemmas 5.1 and 5.2 have the following immediate corollaries. 
Corollary 5.3. If $\alpha: G_{1} \rightarrow G_{2}$ is an epimorphism, then the induced monomorphism $\alpha^{*}: H^{1}\left(G_{2} ; \mathbb{k}\right) \rightarrow H^{1}\left(G_{1} ; \mathbb{k}\right)$ takes $\mathcal{R}^{1}\left(G_{2}, \mathbb{k}\right)$ to $\mathcal{R}^{1}\left(G_{1}, \mathbb{k}\right)$.

In particular, if $G_{1} \cong G_{2}$, there is a linear isomorphism of ambient affine spaces, restricting to an isomorphism $\mathcal{R}^{1}\left(G_{1}, \mathbb{k}\right) \cong \mathcal{R}^{1}\left(G_{2}, \mathbb{k}\right)$. In other words, the ambient linear isomorphism type of the resonance variety is an invariant of the group.

Corollary 5.4. Suppose $G=G_{1} * G_{2}$ splits as a nontrivial free product of subgroups $G_{1}$ and $G_{2}$, with $H^{1}\left(G_{i} ; \mathbb{k}\right) \neq 0$. Then $\mathcal{R}^{1}(G, \mathbb{k})=H^{1}(G ; \mathbb{k})$.

5.3. Resonance of right-angled Artin groups. Let $\Gamma=(\mathrm{V}, \mathrm{E})$ be a finite graph, and let $G_{\Gamma}$ be the corresponding right-angled Artin group. Fix a field $\mathbb{k}$. Write $H_{\mathbb{V}}=$ $H^{1}\left(G_{\Gamma} ; \mathbb{k}\right)$ for the $\mathbb{k}$-vector space with basis canonically indexed by $\mathrm{V}$. If $\mathrm{W}$ is a subset of $\mathrm{V}$, write $H_{\mathrm{W}}$ for the coordinate subspace spanned by $\mathrm{W}$.

Theorem 5.5. Let $\Gamma=(\mathrm{V}, \mathrm{E})$ be a finite graph. Then:

$$
\mathcal{R}^{1}\left(G_{\Gamma}, \mathbb{k}\right)=\bigcup_{\substack{\mathrm{W} \subset \mathrm{V} \\ \Gamma_{\mathrm{W}} \text { disconnected }}} H_{\mathrm{W}} .
$$

Proof. Let $U$ be the union of coordinate subspaces on the right side of (5.5). We need to show $\mathcal{R}^{1}\left(G_{\Gamma}, \mathbb{k}\right)=U$. For an element $a=\sum_{v \in \mathrm{V}} a_{v} v$ in $H_{\mathrm{V}}$, we will write $\operatorname{supp}(a)=\left\{v \in \mathrm{V} \mid a_{v} \neq 0\right\}$ for its support.

Let $a \in U$. Then there is a subset $\mathrm{W} \subset \mathrm{V}$ such that $\operatorname{supp}(a) \subset \mathrm{W}$ and the graph $\Gamma_{\mathrm{W}}$ is disconnected. (Since $0 \in \mathcal{R}^{1}\left(G_{\Gamma}, \mathbb{k}\right)$, we may assume $a \neq 0$, and so $\operatorname{supp}(a) \neq \emptyset$.) Since $\operatorname{supp}(a) \subset \mathrm{W}$, we have $a \in H_{\mathrm{W}}$. Since $\Gamma_{\mathrm{W}}$ is disconnected, $\mathcal{R}^{1}\left(G_{\mathrm{W}}, \mathbb{k}\right)=H_{\mathrm{W}}$, by Corollary [5.4. Thus, $a \in \mathcal{R}^{1}\left(G_{\mathrm{W}}, \mathbb{k}\right)$. Now, Corollary [5.3] applied to the natural epimorphism $G_{\Gamma} \rightarrow G_{\mathrm{W}}$, shows that $\mathcal{R}^{1}\left(G_{\mathrm{W}}, \mathbb{k}\right) \subset \mathcal{R}^{1}\left(G_{\Gamma}, \mathbb{k}\right)$. Hence, $a \in \mathcal{R}^{1}\left(G_{\Gamma}, \mathbb{k}\right)$.

Let $a \notin U$. Then, for every subset $\mathrm{W} \subset \mathrm{V}$ containing $\operatorname{supp}(a)$, the graph $\Gamma_{\mathrm{W}}$ is connected. We need to show $a \notin \mathcal{R}^{1}\left(G_{\Gamma}, \mathbb{k}\right)$, i.e., if there is $a^{\prime}=\sum_{v \in \mathrm{V}} a_{v}^{\prime} v \in H_{\mathrm{\vee}}$ such that $\mu\left(a \wedge a^{\prime}\right)=0$, then $a \wedge a^{\prime}=0$.

Fix a linear order on $\mathrm{V}$. Choose as basis for $H^{2}\left(G_{\Gamma}, \mathbb{k}\right)$ the set of oriented edges $e=(v, w)$ with $v<w$. With these choices, the multiplication $\mu: H_{\bigvee} \wedge H_{\vee} \rightarrow H^{2}\left(G_{\Gamma}, \mathbb{k}\right)$ is given by

$$
a \wedge a^{\prime}=\sum_{v<w}\left|\begin{array}{cc}
a_{v} & a_{w} \\
a_{v}^{\prime} & a_{w}^{\prime}
\end{array}\right| v \wedge w \mapsto \mu\left(a \wedge a^{\prime}\right)=\sum_{e=(v, w) \in \mathrm{E}}\left|\begin{array}{cc}
a_{v} & a_{w} \\
a_{v}^{\prime} & a_{w}^{\prime}
\end{array}\right| e .
$$

The condition that $\mu\left(a \wedge a^{\prime}\right)=0$ is thus equivalent to

$$
\left|\begin{array}{ll}
a_{v} & a_{w} \\
a_{v}^{\prime} & a_{w}^{\prime}
\end{array}\right|=0, \quad \text { for all }\{v, w\} \in \mathrm{E} .
$$

To show that $a \wedge a^{\prime}=0$, it is enough to prove the following.

Claim. Fix $w \in \operatorname{supp}(a)$. Then $a_{v}^{\prime}=\frac{a_{w}^{\prime}}{a_{w}} a_{v}$, for all $v \in \mathrm{V}$. 
Let $v \in \mathrm{V}$. First suppose $v \in \operatorname{supp}(a)$. By assumption, the full subgraph on $\operatorname{supp}(a)$ is connected. So pick a path $w=v_{0}, v_{1}, \ldots, v_{k}=v$ in $\operatorname{supp}(a)$. Using condition (5.7) for each edge of this path, we find $a_{v}^{\prime}=\frac{a_{v_{k-1}}^{\prime}}{a_{v_{k-1}}} a_{v}=\cdots=\frac{a_{v_{0}}^{\prime}}{a_{v_{0}}} a_{v}$, as claimed.

Now suppose $v \notin \operatorname{supp}(a)$. Then, by assumption, the full subgraph on $\operatorname{supp}(a) \cup\{v\}$ is connected. Hence, $\operatorname{dist}(v, \operatorname{supp}(a))=1$, that is, there is a vertex $u \in \operatorname{supp}(a)$ such that $\{u, v\} \in \mathrm{E}$. Condition (5.7) on this edge implies $a_{u} a_{v}^{\prime}-a_{v} a_{u}^{\prime}=0$. Since $a_{v}=0$ and $a_{u} \neq 0$, it follows that $a_{v}^{\prime}=0$, as claimed.

Corollary 5.6. Let $\Gamma=(\mathrm{V}, \mathrm{E})$ be a finite graph, and let $\mathcal{R}^{1}\left(G_{\Gamma}, \mathbb{k}\right) \subset H_{\vee}$ be the resonance variety of the group $G_{\Gamma}$. Then:

(1) The irreducible components of $\mathcal{R}^{1}\left(G_{\Gamma}, \mathbb{k}\right)$ are the coordinate subspaces $H_{\mathrm{W}}$, maximal among those for which $\Gamma_{\mathrm{W}}$ is disconnected.

(2) The codimension of $\mathcal{R}^{1}\left(G_{\Gamma}, \mathbb{k}\right)$ equals the connectivity of $\Gamma$.

In particular, if $\Gamma$ is disconnected, then $\mathcal{R}^{1}\left(G_{\Gamma}, \mathbb{k}\right)=H_{\mathrm{V}}$.

Remark 5.7. Right-angled Artin groups share some common features with arrangement groups, that is, fundamental groups of complements of finite unions of hyperplanes in complex affine space. Indeed, if $G$ is a group in either class, then $G$ admits a finite presentation, with commutator relators only; it is 1-formal; and the resonance variety $\mathcal{R}^{1}(G, \mathbb{C})$ is a union of linear subspaces. However, the intersection of these two classes of groups is fairly small. Indeed, a right-angled Artin group $G_{\Gamma}$ is an arrangement group if and only if $\Gamma$ is a complete multipartite graph. This assertion will be proved elsewhere, in a more general context. Let us only note here that a necessary condition for a group $G$ to be an arrangement group is that any two distinct components of $\mathcal{R}^{1}(G, \mathbb{C})$ intersect only at the origin, see 19. This condition is violated by most graphs we will discuss in 86 for instance, circuits of length at least 5 .

5.4. Bieri-Neumann-Strebel invariants. Let $G$ be a finitely generated group. Pick a finite generating set for $G$, and let $\mathcal{C}(G)$ be the corresponding Cayley graph. Given an additive real character $\chi: G \rightarrow \mathbb{R}$, let $\mathcal{C}_{\chi}(G)$ be the full subgraph on vertex set $\{g \in G \mid \chi(g) \geq 0\}$. In [4], Bieri, Neumann, and Strebel define the (first) BNS invariant of $G$ to be:

$$
\Sigma^{1}(G)=\left\{\chi \in \operatorname{Hom}(G, \mathbb{R}) \backslash\{0\} \mid \mathcal{C}_{\chi}(G) \text { is connected }\right\} .
$$

Clearly, $\Sigma^{1}(G)$ is a conical subset of the vector space $\operatorname{Hom}(G, \mathbb{R})=H^{1}(G ; \mathbb{R})$. It turns out that $\Sigma^{1}(G)$ does not depend on the choice of generating set for $G$, see 4 .

The BNS invariants of right-angled Artin groups have been described in detail by Meier, Meinert, and VanWyk [20, 21]. Using these descriptions, together with our results above, we can identify the set $\Sigma^{1}\left(G_{\Gamma}\right)$ in terms of the resonance variety $\mathcal{R}^{1}\left(G_{\Gamma}, \mathbb{R}\right)$.

Proposition 5.8. Let $G_{\Gamma}$ be a right-angled Artin group.

(1) $\Sigma^{1}\left(G_{\Gamma}\right)=H^{1}\left(G_{\Gamma} ; \mathbb{R}\right) \backslash \mathcal{R}^{1}\left(G_{\Gamma}, \mathbb{R}\right)$.

(2) Suppose $\chi: G_{\Gamma} \rightarrow \mathbb{R}$ is a rational character, i.e., $\operatorname{im}(\chi) \cong \mathbb{Z}$. Then $\operatorname{ker}(\chi)$ is finitely generated if and only if $\chi \notin \mathcal{R}^{1}\left(G_{\Gamma}, \mathbb{R}\right)$. 
Proof. For a character $\chi: G_{\Gamma} \rightarrow \mathbb{R}$, let $\operatorname{supp}(\chi)=\left\{v \in \mathrm{V}_{\Gamma} \mid \chi(v) \neq 0\right\}$ be its support. Theorem [5.5] can be restated, as follows: $\chi$ is not in $\mathcal{R}^{1}\left(G_{\Gamma}, \mathbb{R}\right)$ if and only if, for any $\mathrm{W} \subset \mathrm{V}_{\Gamma}$ with $\operatorname{supp}(\chi) \subset \mathrm{W}$, the graph $\Gamma_{\mathrm{W}}$ is connected.

In [21], Meier and VanWyk show that a character $\chi: G_{\Gamma} \rightarrow \mathbb{R}$ belongs to $\Sigma^{1}\left(G_{\Gamma}\right)$ if and only if the full subgraph on $\operatorname{supp}(\chi)$ is connected and dominant. (A subgraph $\Gamma^{\prime} \subset \Gamma$ is dominant if any vertex $v \in \mathrm{V}_{\Gamma} \backslash \mathrm{V}_{\Gamma^{\prime}}$ has a neighbor in $\Gamma^{\prime}$.)

Comparing these two characterizations finishes the proof of Part (II).

To prove Part (2), we need the following basic result from [4], concerning rational characters $\chi: G \rightarrow \mathbb{R}$ : the group $\operatorname{ker}(\chi)$ is finitely generated if and only if both $\chi$ and $-\chi$ belong to $\Sigma^{1}(G)$. On the other hand, it was shown in 21] that $\Sigma^{1}\left(G_{\Gamma}\right)=-\Sigma^{1}\left(G_{\Gamma}\right)$. Using these two facts, Part (2) follows from Part (10).

A generalization to higher $\Sigma$-invariants and higher resonance varieties will be given in a forthcoming paper.

\section{EXAMPLES}

We now illustrate with a few examples how to compute the LCS ranks, the Chen ranks, and the resonance varieties of right-angled Artin groups, using the results from the three previous sections. We start by discussing methods for computing the two graph polynomials that appear in formulas (3.5) and (4.5).

6.1. Computing the clique and cut polynomials. When a graph $\Gamma$ has no triangles (i.e., when $\Delta_{\Gamma}=\Gamma$ ), the clique polynomial is easy to compute: $P_{\Gamma}(t)=1+\left|\mathrm{V}_{\Gamma}\right| t+$ $\left|\mathrm{E}_{\Gamma}\right| t^{2}$. For an arbitrary graph, the computation of $P_{\Gamma}(t)$ is more complicated, but a simple recursion formula is readily available.

Fix an edge $e \in \mathrm{E}_{\Gamma}$. Let $\Gamma \backslash e$ be the graph $\Gamma$ with edge $e$ removed, and let $\Gamma \backslash N(e)$ be the graph obtained from $\Gamma$ by removing the endpoints of $e$, all their neighbors, and all edges containing any one of these vertices. Using (4.1) and Theorem 5.1 from [28], we find:

$$
P_{\Gamma}(t)=P_{\Gamma \backslash e}(t)-t^{2} P_{\Gamma \backslash N(e)}(t) .
$$

Next, we give some (partial) recursion formulas for the coefficients of the cut polynomial. An edge $e \in \mathrm{E}_{\Gamma}$ is called a bridge if it does not lie in any cycle; we say $e$ is a near-bridge if the only cycles containing $e$ are those incident on all vertices of $\Gamma$.

Lemma 6.1. Suppose the graph $\Gamma$ has a near-bridge e. Then, for all $j<\left|\vee_{\Gamma}\right|$,

$$
c_{j}(\Gamma)=c_{j}(\Gamma \backslash e)-\left(\begin{array}{c}
\left|\mathrm{V}_{\Gamma}\right|-2 \\
j-2
\end{array}\right) .
$$


Proof. Put $\Gamma^{\prime}=\Gamma \backslash e$. Fix a proper subset $\mathrm{W} \subset \mathrm{V}$. If $e \not \subset \mathrm{W}$, then clearly $\tilde{b}_{0}\left(\Gamma_{\mathrm{W}}\right)=$ $\tilde{b}_{0}\left(\Gamma_{\mathrm{W}}^{\prime}\right)$. If $e \subset \mathrm{W}$, then, by the assumption on $e$, we have $\tilde{b}_{0}\left(\Gamma_{\mathrm{W}}\right)=\tilde{b}_{0}\left(\Gamma_{\mathrm{W}}^{\prime}\right)-1$. Hence:

$$
\begin{aligned}
c_{j}(\Gamma) & =\sum_{|\mathrm{W}|=j, e \not \subset \mathrm{W}} \tilde{b}_{0}\left(\Gamma_{\mathrm{W}}\right)+\sum_{|\mathrm{W}|=j, e \subset \mathrm{W}} \tilde{b}_{0}\left(\Gamma_{\mathrm{W}}\right) \\
& =\sum_{|\mathrm{W}|=j, e \not \subset \mathrm{W}} \tilde{b}_{0}\left(\Gamma_{\mathrm{W}}^{\prime}\right)+\sum_{|\mathrm{W}|=j, e \subset \mathrm{W}}\left(\tilde{b}_{0}\left(\Gamma_{\mathrm{W}}^{\prime}\right)-1\right),
\end{aligned}
$$

from which (6.2) follows at once.

Lemma 6.2. Let $\Gamma=\Gamma^{\prime} \coprod K_{1}$ be the disjoint union of a graph $\Gamma^{\prime}$ with a singleton graph $K_{1}$. Then, for all $j \geq 2$ :

$$
c_{j}(\Gamma)=c_{j}\left(\Gamma^{\prime}\right)+c_{j-1}\left(\Gamma^{\prime}\right)+\left(\begin{array}{c}
\left|\mathrm{V}_{\Gamma}\right|-1 \\
j-1
\end{array}\right) .
$$

Proof. We have:

$$
\begin{aligned}
c_{j}(\Gamma) & =\sum_{|\mathrm{W}|=j, \mathrm{~W} \subset \mathrm{V}_{\Gamma^{\prime}}} \tilde{b}_{0}(\Gamma \mathrm{W})+\sum_{|\mathrm{W}|=j, \mathrm{~W} \not \subset \mathrm{V}_{\Gamma^{\prime}}} \tilde{b}_{0}\left(\Gamma_{\mathrm{W}}\right) \\
& =\sum_{|\mathrm{W}|=j, \mathrm{~W} \subset \mathrm{V}_{\Gamma^{\prime}}} \tilde{b}_{0}\left(\Gamma_{\mathrm{W}}^{\prime}\right)+\sum_{|\mathrm{W}|=j-1, \mathrm{~W} \subset \mathrm{V}_{\Gamma^{\prime}}}\left(\tilde{b}_{0}\left(\Gamma_{\mathrm{W}}^{\prime}\right)+1\right),
\end{aligned}
$$

from which (6.3) follows at once.

6.2. Trees. Let $\Gamma$ be a tree on $n$ vertices. In this case, $\Delta_{\Gamma}=\Gamma$, and so the clique polynomial is $P_{\Gamma}(t)=1+n t+(n-1) t^{2}$. Hence, by Theorem 3.4(4),

$$
\prod_{k=1}^{\infty}\left(1-t^{k}\right)^{\phi_{k}\left(G_{\Gamma}\right)}=(1-t)(1-(n-1) t) .
$$

We claim the cut numbers of $\Gamma$ are given by

$$
c_{j}(\Gamma)=(j-1)\left(\begin{array}{c}
n-1 \\
j
\end{array}\right), \quad \text { for } j=2, \ldots, n-1 .
$$

For $n=1$ or 2 , there is nothing to prove. Let $e$ be an extremal edge. Clearly, $e$ is a bridge, and $\Gamma \backslash e=\Gamma_{0} \coprod K_{1}$, where $\Gamma_{0}$ is a tree on $n-1$ vertices. Formula (6.5) follows by induction on $n$ (starting from $n=3$, where it is obvious), using Lemmas 6.1 and 6.2 .

Before proceeding, let us note that (6.5) is equivalent to $\beta_{i, i+1}^{S}\left(S / I_{\Gamma}\right)=i\left(\begin{array}{c}n-1 \\ i+1\end{array}\right)$, for $1 \leq i \leq n-2$, a fact which can also be deduced from [11, Proposition 11].

Using Theorem 4.1(3) and formula (6.5), we find that the Chen ranks of $G_{\Gamma}$ are given by $\theta_{k}\left(G_{\Gamma}\right)=(k-1) \cdot\left(\begin{array}{c}k+n-3 \\ k\end{array}\right)$, for all $k \geq 2$, or, equivalently:

$$
\sum_{k=2}^{\infty} \theta_{k}\left(G_{\Gamma}\right) t^{k}=1-\frac{1-(n-1) t}{(1-t)^{n-1}} .
$$


Now consider the resonance variety $\mathcal{R}^{1}\left(G_{\Gamma}, \mathbb{k}\right)$. The minimal cutsets of the tree $\Gamma$ are the non-extremal vertices. Thus, $\mathcal{R}^{1}\left(G_{\Gamma}, \mathbb{k}\right)$ consists of coordinate hyperplanes in $\mathbb{k}^{n}$, one for each non-extremal vertex:

$$
\mathcal{R}^{1}\left(G_{\Gamma}, \mathbb{k}\right)=\bigcup_{v \in \mathrm{V}: v \text { non-extremal }} H_{\bigvee \backslash\{v\}}
$$

The above discussion shows that, for a tree $\Gamma$, the LCS ranks and the Chen ranks of $G_{\Gamma}$ depend only on $n=\left|V_{\Gamma}\right|$, whereas the resonance variety $\mathcal{R}^{1}\left(G_{\Gamma}, \mathbb{k}\right)$ also depends on the cut sets of the graph. We illustrate this phenomenon with an example.

Example 6.3. Let $\Gamma$ and $\Gamma^{\prime}$ be two trees on the same vertex set, but with different number of extremal vertices. (For example, take the Dynkin graphs $\Gamma=\mathrm{A}_{n}$ and $\Gamma^{\prime}=$ $\mathrm{D}_{n}$, with $n \geq 4$; clearly, $\Gamma$ has 2 extremal vertices, whereas $\Gamma^{\prime}$ has 3 such vertices.) Then $\phi_{k}\left(G_{\Gamma}\right)=\phi_{k}\left(G_{\Gamma^{\prime}}\right)$ and $\theta_{k}\left(G_{\Gamma}\right)=\theta_{k}\left(G_{\Gamma^{\prime}}\right)$, for all $k \geq 1$, yet $\mathcal{R}^{1}\left(G_{\Gamma}, \mathbb{k}\right) \neq \mathcal{R}^{1}\left(G_{\Gamma^{\prime}}, \mathbb{k}\right)$. Hence, $\mathfrak{H}\left(G_{\mathrm{A}_{n}}\right)$ and $\mathfrak{H}\left(G_{\mathrm{D}_{n}}\right)$ are not isomorphic as graded Lie algebras, although they have the same graded ranks.

6.3. Circuits. Let $\Gamma_{n}$ be a circuit on $n$ vertices, $n \geq 4$. Again, $\Delta_{\Gamma_{n}}=\Gamma_{n}$, and so $P_{\Gamma_{n}}(t)=1+n t+n t^{2}$. Hence,

$$
\prod_{k=1}^{\infty}\left(1-t^{k}\right)^{\phi_{k}\left(G_{\Gamma_{n}}\right)}=1-n t+n t^{2} .
$$

If $n \geq 5$, the right side of (6.8) does not factor into linear factors in $\mathbb{Z}[t]$, and so the group $G_{\Gamma_{n}}$ cannot decompose as an (iterated) semi-direct product of free groups, with trivial monodromy action in homology.

Deleting an edge from $\Gamma_{n}$ yields the $n$-chain $\mathrm{A}_{n}$. Clearly, any edge in $\Gamma_{n}$ is a nearbridge; thus, by Lemma 6.1 $c_{j}\left(\Gamma_{n}\right)=c_{j}\left(\mathrm{~A}_{n}\right)-\left(\begin{array}{c}n-2 \\ j-2\end{array}\right)$, for all $j<n$. On the other hand, $c_{n}\left(\Gamma_{n}\right)=0$, since $\Gamma_{n}$ is connected. Using formula (6.5), and plugging into (4.5) gives:

$$
\sum_{k=2}^{\infty} \theta_{k}\left(G_{\Gamma_{n}}\right) t^{k}=\sum_{j=2}^{n-2}\left((j-1)\left(\begin{array}{c}
n-1 \\
j
\end{array}\right)-\left(\begin{array}{c}
n-2 \\
j-2
\end{array}\right)\right)\left(\frac{t}{1-t}\right)^{j} .
$$

The resonance variety $\mathcal{R}^{1}\left(G_{\Gamma_{n}}, \mathbb{k}\right)$ consists of $n(n-3) / 2$ codimension 2 subspaces in $\mathbb{k}^{n}$, one for each pair of non-adjacent vertices:

$$
\mathcal{R}^{1}\left(G_{\Gamma_{n}}, \mathbb{k}\right)=\bigcup_{\{v, w\} \in \mathrm{E}_{\overline{\Gamma_{n}}}} H_{\bigvee \backslash\{v, w\}}
$$

Example 6.4. For each $n \geq 5$, let $\Gamma_{n}^{\prime}$ be an $(n-1)$-circuit, with an extra vertex and an edge attaching it to the circuit. Clearly, $P_{\Gamma_{n}^{\prime}}(t)=P_{\Gamma_{n}}(t)$, and so $\phi_{k}\left(G_{\Gamma_{n}^{\prime}}\right)=\phi_{k}\left(G_{\Gamma_{n}}\right)$, for all $k \geq 1$.

Although the LCS ranks of $G_{\Gamma_{n}^{\prime}}$ and $G_{\Gamma_{n}}$ are the same, the Chen ranks are different. Indeed, an application of Lemmas 6.1 and 6.2 yields $c_{j}\left(\Gamma_{n}^{\prime}\right)=c_{j}\left(\Gamma_{n}\right)$ for $j=2, \ldots, n-2$. 

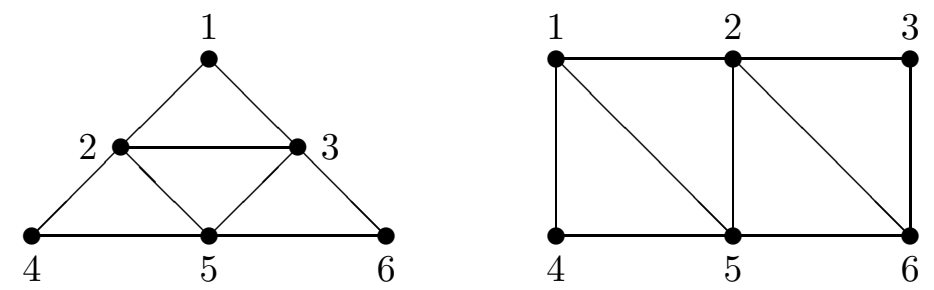

Figure 1. The graphs $\Gamma$ and $\Gamma^{\prime}$

On the other hand, $c_{n-1}\left(\Gamma_{n}^{\prime}\right)=1$, whereas $c_{n-1}\left(\Gamma_{n}\right)=0$. Thus,

$$
\sum_{k=2}^{\infty} \theta_{k}\left(G_{\Gamma_{n}^{\prime}}\right) t^{k}=\sum_{j=2}^{n-2}\left((j-1)\left(\begin{array}{c}
n-1 \\
j
\end{array}\right)-\left(\begin{array}{c}
n-2 \\
j-2
\end{array}\right)\right)\left(\frac{t}{1-t}\right)^{j}+\left(\frac{t}{1-t}\right)^{n-1} .
$$

Consequently, $\theta_{k}\left(G_{\Gamma_{n}^{\prime}}\right)=\theta_{k}\left(G_{\Gamma_{n}}\right)$ for $k \leq n-2$, but $\theta_{k}\left(G_{\Gamma_{n}^{\prime}}\right)>\theta_{k}\left(G_{\Gamma_{n}}\right)$, for $k \geq n-1$.

6.4. A pair of graphs. We conclude this section with a pair of right-angled Artin groups that differ in a rather subtle way.

Example 6.5. Let $\Gamma$ and $\Gamma^{\prime}$ be the two graphs in Figure 11. Both graphs have clique polynomial $P(t)=1+6 t+9 t^{2}+4 t^{3}$ and cut polynomial $Q(t)=t^{2}\left(6+8 t+3 t^{2}\right)$; thus, $G_{\Gamma}$ and $G_{\Gamma^{\prime}}$ have the same LCS and Chen ranks. Their resonance varieties have the same number of irreducible components, each a codimension 2 linear subspace:

$$
\mathcal{R}^{1}\left(G_{\Gamma}, \mathbb{k}\right)=H_{\overline{23}} \cup H_{\overline{25}} \cup H_{\overline{35}}, \quad \mathcal{R}^{1}\left(G_{\Gamma^{\prime}}, \mathbb{k}\right)=H_{\overline{15}} \cup H_{\overline{25}} \cup H_{\overline{26}} .
$$

Yet the two varieties are not isomorphic, since $\operatorname{dim}\left(H_{\overline{23}} \cap H_{\overline{25}} \cap H_{\overline{35}}\right)=3$, whereas $\operatorname{dim}\left(H_{\overline{15}} \cap H_{\overline{25}} \cap H_{\overline{26}}\right)=2$.

\section{Higher-Dimensional CUBical COMPleXes}

The cubical complex $K_{\Gamma}$ associated to a graph $\Gamma$ has natural, higher-dimensional analogues, introduced in [18]. For each integer $q \geq 0$, let $K_{\Gamma}^{q}$ be the CW-complex obtained from $\left(S^{2 q+1}\right)^{\times n}, n=\left|\mathrm{V}_{\Gamma}\right|$, by deleting the cells corresponding to the non-faces of the flag complex $\Delta_{\Gamma}$. Note that $K_{\Gamma}^{0}=K_{\Gamma}=K\left(G_{\Gamma}, 1\right)$, while $K_{\Gamma}^{q}$ is simply-connected, for $q \geq 1$.

Let $\Omega Y$ denote the based loop space of a pointed CW-complex $Y$. In [18, Kim and Roush determined the Pontryagin ring structure of $H_{*}\left(\Omega K_{\Gamma}^{q}\right)$. We now pursue this study, and determine the natural Hopf algebra structure on $H_{*}\left(\Omega K_{\Gamma}^{q} ; \mathbb{Q}\right)$. As a byproduct, we compute explicitly the rational homology and homotopy groups of $\Omega K_{\Gamma}^{q}$, in terms of the clique polynomial of $\Gamma$.

First, some background. The homotopy Lie algebra of a simply-connected, finite-type $\mathrm{CW}$-complex $Y$ is the graded $\mathbb{Q}$-vector space

$$
\pi_{*}(\Omega Y) \otimes \mathbb{Q}=\bigoplus_{k \geq 1} \pi_{k}(\Omega Y) \otimes \mathbb{Q}
$$


with the graded Lie algebra structure coming from the Whitehead product. By the Milnor-Moore theorem 22], the universal enveloping algebra of $\pi_{*}(\Omega Y) \otimes \mathbb{Q}$ is isomorphic, as a Hopf algebra, to $H_{*}(\Omega Y, \mathbb{Q})$. Finally, we say $Y$ is coformal if its rational homotopy type is determined by its homotopy Lie algebra.

Theorem 7.1. Let $\Gamma=(\mathrm{V}, \mathrm{E})$ be a finite graph, and $q$ a positive integer. Then:

(1) The homotopy Lie algebra of $K_{\Gamma}^{q}$ has presentation

$$
\pi_{*}\left(\Omega K_{\Gamma}^{q}\right) \otimes \mathbb{Q}=\operatorname{Lie}(\mathrm{V}[q]) /([v, w]=0 \text { if }\{v, w\} \in \mathrm{E}),
$$

where $\mathrm{Lie}(\mathrm{V}[q])$ denotes the free Lie algebra on generators from $\mathrm{V}$, in degree $2 q$.

(2) The ranks of the graded pieces, $\Phi_{k}\left(K_{\Gamma}^{q}\right)=\operatorname{rank} \pi_{k}\left(\Omega K_{\Gamma}^{q}\right)$, vanish if $2 q \nmid k$, while the other ranks are given by the following homotopy LCS formula:

$$
\prod_{k=1}^{\infty}\left(1-t^{(2 q+1) k}\right)^{\Phi_{2 q k}\left(K_{\Gamma}^{q}\right)}=P_{\Gamma}\left(-t^{2 q+1}\right) .
$$

(3) The Hilbert series of $H_{*}\left(\Omega K_{\Gamma}^{q} ; \mathbb{Q}\right)$ is given by

$$
\operatorname{Poin}\left(\Omega K_{\Gamma}^{q}, t\right)=P_{\Gamma}\left(-t^{2 q}\right)^{-1} .
$$

(4) If $2 q+1>\operatorname{dim} K_{\Gamma}$, then $K_{\Gamma}^{q}$ is both formal and coformal.

Proof. Let $X=K_{\Gamma}$ and $Y=K_{\Gamma}^{q}$. Following [18, note that the ring $H^{*}(Y)$ is the $q$-rescaling of the ring $H^{*}(X)$, with rescaling factor of $2 q+1$; in particular,

$$
\operatorname{Poin}(Y, t)=\operatorname{Poin}\left(X, t^{2 q+1}\right)=P_{\Gamma}\left(t^{2 q+1}\right) .
$$

Now recall that $H^{*}(X ; \mathbb{Q})$ is a Koszul algebra. Therefore, by [24, Theorem B], the graded Lie algebra $\pi_{*}(\Omega Y) \otimes \mathbb{Q}$ is the $q$-rescaling of $\operatorname{gr}\left(G_{\Gamma}\right) \otimes \mathbb{Q}$, with rescaling factor of 2q. Part (11) follows from Theorem 3.4, Parts (11) and (31).

Parts (21) and (3) are direct consequences of Theorem $\mathrm{C}$ and formula (28) from [24].

As for Part (4), formality is guaranteed by [24, $\S 4.1$ and Proposition 4.4], while coformality follows from [24, Proposition 1.12].

Remark 7.2. The conclusion of Theorem 7.1 may not hold for an arbitrary subcomplex of $\left(S^{2 q+1}\right)^{\times n}$. Indeed, let $Y$ be the $(2 q+1)(n-1)$-skeleton of $\left(S^{2 q+1}\right)^{\times n}$. Then $Y$ is formal. Nevertheless, the vanishing property from Part (2) and the coformality property from Part (4) fail for $Y$, as soon as $2 q+1>n-1>1$; see [24, Example 8.5].

Example 7.3. Let $\Gamma$ and $\Gamma^{\prime}$ be two trees on the same vertex set, but with different number of extremal vertices, e.g., $\Gamma=\mathrm{A}_{n}$ and $\Gamma^{\prime}=\mathrm{D}_{n}$, with $n \geq 4$. Let $Y=K_{\Gamma}^{q}$ and $Y^{\prime}=K_{\Gamma^{\prime}}^{q}$ be the corresponding higher cubical complexes. Then $Y$ and $Y^{\prime}$ are both formal and coformal spaces, by Theorem 7.1(4), and share the same Poincaré polynomial, by formula (7.1); moreover, the rational homology and homotopy groups of $\Omega Y$ and $\Omega Y^{\prime}$ have the same dimensions, by Theorem [7.1(3) and (2). Nevertheless, $Y$ and $Y^{\prime}$ are not (rationally) homotopy equivalent. Indeed, by the computation from Example 6.3. $\mathfrak{H}\left(G_{\Gamma}\right) \neq \mathfrak{H}\left(G_{\Gamma^{\prime}}\right)$. Comparing the Lie algebra presentations from Theorems 3.4 and 7.1, we conclude that $\pi_{*}(\Omega Y) \otimes \mathbb{Q} \neq \pi_{*}\left(\Omega Y^{\prime}\right) \otimes \mathbb{Q}$. 
Added in Proof. After completing this paper, we became aware of the work of Duchamp and Krob 6], 7]. Using Theorem 3.4(1), it is readily seen that Theorem 2.1 of [7] is equivalent to our Theorem 3.4(3), while Corollary II.16 and Theorem III.3 of [6] are equivalent to our Theorem [3.4(2) and (4), respectively. Thus, our methods give a completely different (and much shorter) proof of Duchamp and Krob's results.

Acknowledgments. Computations were carried out with the help of GAP 4.4 13 and Macaulay 2 14.

This work was done while the authors were attending the program "Hyperplane Arrangements and Applications" at the Mathematical Sciences Research Institute in Berkeley, California, in Fall, 2004. We thank MSRI for its support and hospitality during this stay.

\section{REFERENCES}

[1] A. Aramova, L. Avramov, J. Herzog, Resolutions of monomial ideals and cohomology over exterior algebras, Trans. Amer. Math. Soc. 352 (1999), 579-594. MR 2000c:13021

[2] A. Aramova, J. Herzog, T. Hibi, Gotzmann theorems for exterior algebras and combinatorics, Journal of Algebra 191 (1997), 174-211. MR 98c:13025

[3] M. Bestvina, N. Brady, Morse theory and finiteness properties of groups, Invent. Math. 129 (1997), no. 3, 445-470. MR 98i:20039

[4] R. Bieri, W. Neumann, R. Strebel, A geometric invariant of discrete groups, Invent. Math. 90 (1987), no. 3, 451-477. MR 89b:20108

[5] R. Charney, M. Davis, Finite $K(\pi, 1)$ s for Artin groups, Prospects in topology (Princeton, NJ, 1994), 110-124, Ann. of Math. Stud., Vol. 138, Princeton Univ. Press, Princeton, NJ, 1995. MR 97a:57001

[6] G. Duchamp, D. Krob, The free partially commutative Lie algebra: bases and ranks, Adv. Math. 95 (1992), no. 1, 92-126. MR 93j:17009

[7] _ The lower central series of the free partially commutative group, Semigroup Forum $\mathbf{4 5}$ (1992), no. 3, 385-394. MR 93e:20047

[8] D. Eisenbud, S. Popescu, S. Yuzvinsky, Hyperplane arrangement cohomology and monomials in the exterior algebra, Trans. Amer. Math. Soc. 355 (2003), no. 11, 4365-4383. MR 2004g:52036

[9] M. Falk, Arrangements and cohomology, Ann. Combin. 1 (1997), no. 2, 135-157. MR 99g:52017

[10] R. Fröberg, Determination of a class of Poincaré series, Math. Scand. 37 (1975), no. 1, 29-39. MR 0404254

[11] _ Rings with monomial relations having linear resolutions, J. Pure Appl. Algebra 38 (1985), no. 2-3, 235-241. MR 87b:13020

[12] R. Fröberg, C. Löfwall, Koszul homology and Lie algebras with application to generic forms and points, Homology Homotopy Appl. 4 (2002), no. 2, part 2, 227-258. MR 2003g:13018

[13] The GAP Group, GAP-Groups, Algorithms, and Programming, Version 4.4 (2004); available at http://www.gap-system.org.

[14] D. Grayson, M. Stillman, Macaulay 2: a software system for research in algebraic geometry; available at http://www.math.uiuc.edu/Macaulay2.

[15] S. Halperin, J. Stasheff, Obstructions to homotopy equivalences, Adv. Math. 32 (1979), no. 3, 233-279. MR 80j:55016

[16] M. Hochster, Cohen-Macaulay rings, combinatorics, and simplicial complexes, in: Ring theory, II (Proc. Second Conf., Univ. Oklahoma, Norman, Okla., 1975), pp. 171-223, Lecture Notes in Pure and Appl. Math., Vol. 26, Dekker, New York, 1977. MR 0441987 
[17] M. Kapovich, J. Millson, On representation varieties of Artin groups, projective arrangements and the fundamental groups of smooth complex algebraic varieties, Inst. Hautes Études Sci. Publ. Math. 88 (1998), no. 8, 5-95. MR 2001d:14024

[18] K.H. Kim, F.W. Roush, Homology of certain algebras defined by graphs, J. Pure Appl. Algebra 17 (1980), no. 2, 179-186. MR 82e:05114b

[19] A. Libgober, S. Yuzvinsky, Cohomology of the Orlik-Solomon algebras and local systems, Compositio Math. 21 (2000), no. 3, 337-361. MR 2001j:52032

[20] J. Meier, H. Meinert, L. VanWyk, Higher generation subgroup sets and the $\Sigma$-invariants of graph groups, Comment. Math. Helv. 73 (1998), no. 1, 22-44. MR 99f:57002

[21] J. Meier, L. VanWyk, The Bieri-Neumann-Strebel invariants for graph groups, Proc. London Math. Soc. (3) 71 (1995), no. 2, 263-280. MR 96h:20093

[22] J.W. Milnor, J.C. Moore, On the structure of Hopf algebras, Ann. Math. 81 (1965), 211-264. MR 30 \#4259

[23] S. Papadima, A. Suciu, Chen Lie algebras, International Math. Research Notices 2004, no. 21, 1057-1086. MR 2004m:17043

[24] Homotopy Lie algebras, lower central series, and the Koszul property, Geometry \& Topology 8 (2004), 1079-1125. MR 2005g:55022

[25] _ Algebraic invariants for Bestvina-Brady groups, preprint, 2005.

[26] S. Papadima, S. Yuzvinsky, On rational $K[\pi, 1]$ spaces and Koszul algebras, J. Pure Appl. Alg. 144 (1999), no. 2, 156-167. MR 2000k:55017

[27] D. Quillen, Rational homotopy theory, Ann. of Math. 90 (1969), 205-295. MR 0258031

[28] P. Renteln, The Hilbert series of the face ring of a flag complex, Graphs Combin. 18 (2002), no. 3, 605-619. MR 2003i: 13025

[29] M. Roth, A. Van Tuyl, On the linear strand of an edge ideal, preprint arXiv:math.AC/0411181.

[30] H. Schenck, A. Suciu, Resonance, linear syzygies, Chen groups, and the Bernstein-Gelfand-Gelfand correspondence, to appear in Trans. Amer. Math. Soc., available at arXiv:math. AC/0502438.

[31] B. Shelton, S. Yuzvinsky, Koszul algebras from graphs and hyperplane arrangements, J. London Math. Soc. 56 (1997), no. 3, 477-490. MR 99c:16044

[32] R. Stanley, Combinatorics and commutative algebra, Second edition, Progress in Mathematics, vol. 41, Birkhäuser, Boston, MA, 1996. MR 98h:05001

[33] D. Sullivan, Infinitesimal computations in topology, Inst. Hautes Études Sci. Publ. Math. 47 (1977), 269-331. MR 0646078

Institute of Mathematics of the Academy, P.O. Box 1-764, RO-014700 Bucharest, RoMANIA

E-mail address: Stefan.Papadima@imar.ro

Department of Mathematics, Northeastern University, Boston, MA 02115, USA

E-mail address: a.suciu@neu.edu

$U R L:$ http://www . math. neu.edu/ ${ }^{\sim}$ suciu 